\title{
Influence of the Flux Pinning on Critical Current in HTc Superconductors with Nano-Sized Defects
}

\author{
J. Sosnowski*
}

Electrotechnical Institute 04-703 Warsaw, Pożaryskiego 28, Poland

\begin{abstract}
Performed analysis of the flux pinning on nano-sized centers, allowed to receive new results on the critical current problems in the high temperature oxide superconductors. A new model of the interaction pancake vortices with the nano-sized centers is proposed and comparison of obtained results with others pinning forces approaches is presented. The energy balance approach is applied, which leads to the appearance of the potential barrier determining the pancake vortices movement in the flux creep process. Various initial positions of the captured vortex have been analyzed and variation of the screening currents for these configurations has been considered. Initial calculations of the influence of this effect on the current-voltage characteristics have been performed. The dependence of the critical current on the pinning centers parameters has been determined, as well as comparison with the experimental data presented. Pinning force has been calculated and its temperature dependence, which is in qualitative agreement with experiments. The critical current determines the magnetic induction profiles and therefore also flux trapping, very important parameter from the point of view of application HTc superconductors as permanent magnets. The influence of the critical current and granular structure parameters on the flux trapping in ceramic superconductors is regarded. The influence of the critical current on a.c. losses in second generation HTc tapes with magnetic substrate is briefly discussed too.
\end{abstract}

Keywords: High temperature superconductors, flux pinning, critical current.

\section{INTRODUCTION}

The effect of capturing vortices in HTc superconductors has essential meaning from the point of view of electric current transport. Therefore analysis of this subject is important both from the scientific as well as technical point of view. To this subject indicate a lots of papers devoted to the flux pinning (see for instance [1-4] and references cited here). The present paper on other hand is the continuation of the present author's works [5-8]. While much attention of the scientists is devoted mainly to the critical temperature analysis of the HTc superconductors, flux pinning in these materials also brings new yet unresolved problems in comparison with the classic already low temperature superconductors. One of them is strong magnetic field decrease of the critical current in these materials, which in fact limits real applications of HTc superconductors in electric machines. From other side the layered structure of HTc materials leads to the existence of pancake type vortices, which allows already treat pinning interaction as individual one between pinning center and vortex. We remind that in the classic 3D materials the flux lines multifold captured to the various centers appear, which requires treating this case as a many body problem.

\section{NEW MODEL OF THE PINNING INTERACTION}

High critical temperature oxide superconductors are characterized by unique superconducting parameters, as the critical temperature but also the critical current. An essential

*Address correspondence to this author at the Electrotechnical Institute 04703 Warsaw, Pożaryskiego 28, Poland; Tel: 4822 8123478; Fax: 4822 6157535; E-mail: sosnow@iel.waw.pl

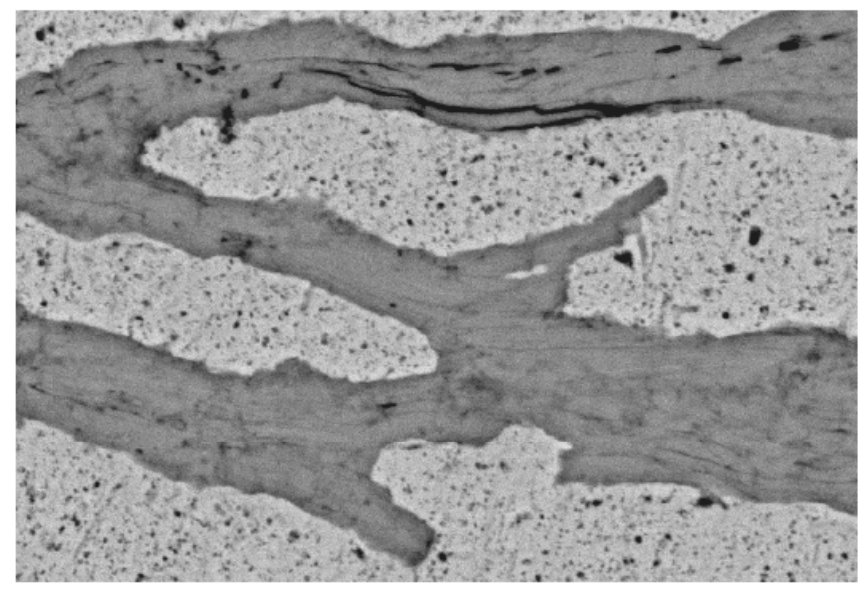

Fig. (1). Scanning electron microscopy (SEM) image of the cross-section, of superconducting Bi-based tape, indicating to the existence of the nanostructural defects (black points - nano-pores and unreacted $\mathrm{CuO}_{2}$ grains). The length of the cross-section is $34 \mu \mathrm{m}$.

property of the high temperature oxide superconducting materials, such as the critical current is very sensitive to the existence of structural defects. These defects called the columnar, if created during the fast neutrons irradiation of the superconducting windings working in nuclear reactors are then of the nanometric size. SEM image of the cross-section of the HTc Bi:2223 tape shown in Fig. (1) indicates just to the existence of nano-dimensional defects in their structure, in the form of nanopores or unreacted $\mathrm{CuO}_{2}$ grains. These defects interact with the pancake vortices arising in HTc superconductors, stabilizing thus the vortex structure and 


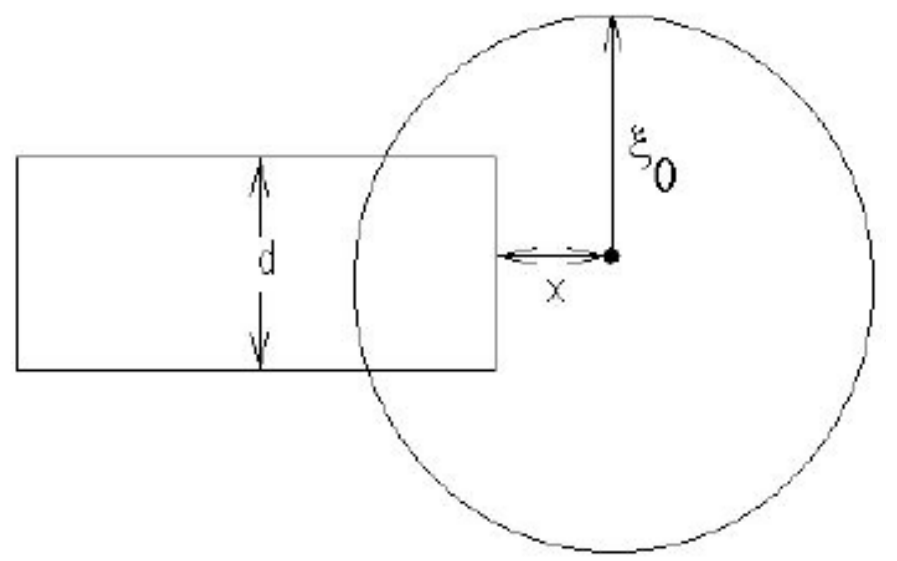

Fig. (2). View of the vortex core of the radius equal to the coherence length $\xi_{0}$ captured on the pinning center of the width $\boldsymbol{d}$

permitting in this way the resistivity-free current flow. For description of this dynamic interaction new model of the vortex capturing has been developed, based on an analysis of the increase in the normal state energy during the pancake vortex deflection from the nano-sized pinning center, against its equilibrium position.

The geometry of this interaction is shown in Fig. (2). The above approximation corresponds roughly to the consideration of the first two terms in the Ginzburg-Landau theory [9]. Two effects have been investigated: of the enhancement in the normal state energy during the vortex movement, as well as the opposite one, of an increase in the elasticity energy of the vortex lattice during the capturing process, deflecting the vortex from its equilibrium position in the magnetic vortices array.

In the initial state, it is for $\mathrm{x}=0$, of the vortex captured on the flat pinning center of nanosized dimension $\boldsymbol{d}$, the normal state energy of vortex core is:

$U_{1}(0)=\frac{\mu_{o} H_{c}^{2} l}{2}\left[\pi \xi^{2}-\xi^{2} \arcsin \frac{d}{2 \xi}-\frac{d \xi}{2} \sqrt{1-\left(\frac{d}{2 \xi}\right)^{2}}\right]$

Parameter $\boldsymbol{\xi}$ shown in Fig. (2) is the coherence length, $\boldsymbol{H}_{\boldsymbol{c}}$ magnetic thermodynamic critical field, $\boldsymbol{l}$ pinning center thickness, $\boldsymbol{\mu}_{\boldsymbol{0}}$ magnetic permeability. Eq. 1 as well as the following relations are received in the geometrical approach, in which vortex core is treated as a cylinder with well defined sharp walls. It means that the square brackets in Eq. 1 and the subsequent ones describe the common part of the geometrical cross-section of the vortex core and pinning center of the perpendicular shape. For a vortex deflected on the distance $\boldsymbol{x}$ against this equilibrium position as shows Fig. (2), the energy potential depth is given as:

$U_{2}(x)=\frac{\mu_{o} H_{c}^{2} l}{2}\left[\pi \xi^{2}+d x-\xi^{2} \arcsin \frac{d}{2 \xi}-\frac{d \xi}{2} \sqrt{1-\left(\frac{d}{2 \xi}\right)^{2}}\right]$

for $\mathrm{x}<\mathrm{x}_{\mathrm{c}}$, where $\boldsymbol{x}_{\boldsymbol{C}}$ is defined as:

$x_{c}=\xi \sqrt{1-\left(\frac{d}{2 \xi}\right)^{2}}$

The deflection of a vortex is caused by the flow of the current in the magnetic field, which leads to an rise of the
Lorentz force, tearing the vortices from the initial captured position. For a vortex deflected for a distance larger than $\boldsymbol{x}_{\boldsymbol{C}}$ the energy of the normal state connected then with shifted vortex is given as follows:

$U_{3}(x)=\frac{\mu_{o} H_{c}^{2} l_{p} \xi^{2}}{2}\left[\frac{\pi}{2}+\arcsin \frac{x}{\xi}+\frac{x}{\xi} \sqrt{1-\left(\frac{x}{\xi}\right)^{2}}\right]$

From relations $1-4$ the value of the potential barrier is determined:

$\Delta U_{2}(x)=\frac{\mu_{o} H_{c}^{2} l d x}{2}$

in the case of $\boldsymbol{x}<\boldsymbol{x}_{\boldsymbol{c}}$, while in the opposite case it is:

$\Delta U_{3}(x)=\frac{\mu_{o} H_{c}^{2} l \xi^{2}}{2}\left[\arcsin \frac{x}{\xi}-\frac{\pi}{2}+\arcsin \frac{d}{2 \xi}+\frac{x}{\xi} \sqrt{1-\left(\frac{x}{\xi}\right)^{2}}+\frac{d}{2 \xi} \sqrt{1-\left(\frac{d}{2 \xi}\right)^{2}}\right]$

The maximum of the potential barrier is reached just in this second case. In the total energy balance additionally has been taken into account the Lorentz force arising during the transport current flow, which affects the movement of the vortices as well as elasticity forces of the vortex array. Elasticity forces are related to the magnitude of the captured vortex deflection in the vortex lattice. Capturing the vortices by the nano-sized pinning centers causes the shift of the vortex from its equilibrium position in the regular vortex array, thus leading to an increase in the elasticity energy of the structure of the vortex lattice. This effect is the function of the deflection of the individual vortex from its equilibrium position in the lattice. We have taken it into account by assuming that an enhancement of the vortex elasticity energy is proportional to the square of the length of the vortex deflection, with coefficient of proportionality expressed by the value of the parameter $\alpha$ :

$U_{e l}=\frac{2 c_{s} \pi \xi^{2}(\xi-x)^{2}}{l_{a}}=\alpha(\xi-x)^{2}$

Parameter $\boldsymbol{c}_{\mathrm{s}}$ is the corresponding elasticity shear modulus, while $\boldsymbol{l}_{\mathrm{a}} \approx \boldsymbol{l}$ denotes the length at which the magnetic flux lines of vortices are deformed. Parameter $\boldsymbol{l}$ as it was stated in Eq. 1 is the pinning center thickness. The above model leads finally to the relation describing the po- 


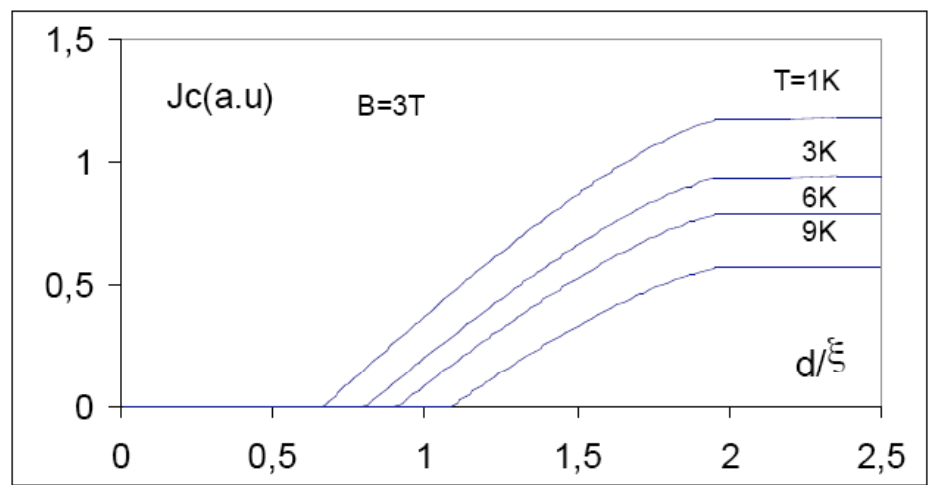

Fig. (3). The dependence of the critical current satisfying the electric field criterion versus the pinning center dimensions for various temperatures, given at each curve. Magnetic induction is constant and equal to 3T.

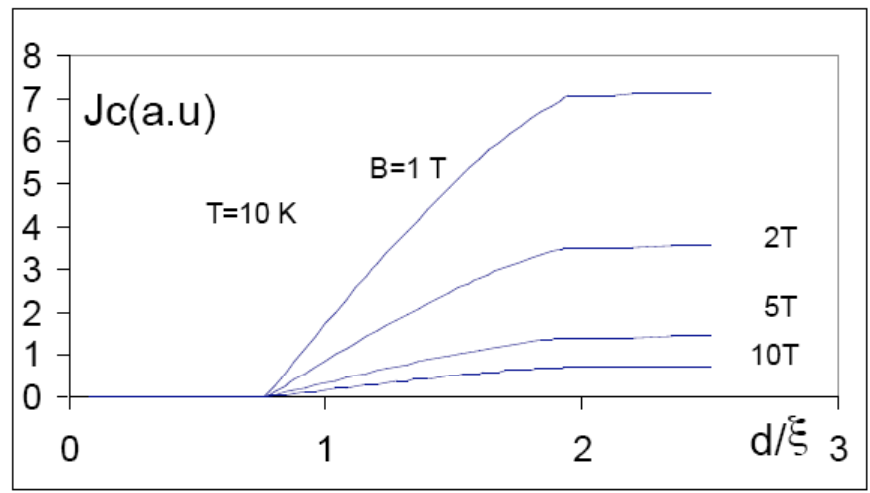

Fig. (4). The influence of the nano-sized pinning centers of the width $\boldsymbol{d}$, normalized to the coherence length $\boldsymbol{\xi}$ on the critical current density versus applied magnetic field.

tential barrier for the flux creep process $\boldsymbol{\Delta} \boldsymbol{U}$ in the function of the reduced current density $\boldsymbol{i}=\boldsymbol{j} / \boldsymbol{j}_{\boldsymbol{C}}$, where $\boldsymbol{j}_{\boldsymbol{C}}$ is defined as the critical transport current density for flux creep process. Condition for the minimum of the system energy allows therefore to deduce the final expression for the energy barrier height, which should be crossed by the vortex in the flux creep process:

$\Delta U(i)=\frac{\mu_{0} H_{c}^{2} l \xi^{2}}{2} \Theta+\alpha \xi^{2} \sqrt{1-i^{2}}\left(\sqrt{1-i^{2}}-2\right)$

Function $\Theta$ appearing in Eq. 8 is determined here according to the relation:

$\Theta=\arcsin \frac{d}{2 \xi}+\frac{d}{2 \xi} \sqrt{1-\left(\frac{d}{2 \xi}\right)^{2}}-i \sqrt{1-i^{2}}-\arcsin i$

Parameter $\boldsymbol{\alpha}$ describes the elasticity energy of the vortex lattice. $j_{C}$ is defined here as the transport current density $\boldsymbol{j}$ for which the potential barrier disappears, in the large scale limit of the pinning center size, it is for $\boldsymbol{d}=\mathbf{2} \boldsymbol{\xi}$, according to the relation:

$$
j_{c}=\frac{\mu_{0} H_{c}^{2}}{\pi \xi B} \cdot \frac{S_{c}\left(1-S_{c} / a^{2}\right)}{a^{2}}
$$

$\boldsymbol{S}_{\boldsymbol{c}}$ in Eq. 10 is the pinning center cross-section, while $\boldsymbol{a}$ is the average distance between the regularly ordered pinning centers and $\boldsymbol{B}$ is magnetic induction.
We insert then the expressions 8-9 into the constitutive relation, describing the generated electric field in the flux creep process, just as a function of the potential barrier height:

$E=-B \omega a\left[\exp \left[-\frac{\Delta U_{0}}{k_{B} T}\left(1+\frac{j}{j_{C}}\right)\right]-\exp \left(-\frac{\Delta U}{k_{B} T}\right)\right]$

$\boldsymbol{\Delta} \boldsymbol{U}_{\boldsymbol{0}}$ is the potential barrier height without current, $\boldsymbol{\omega}$ the constant value describing the characteristic flux creep process frequency, $\boldsymbol{T}$ temperature and $\boldsymbol{k}_{\boldsymbol{B}}$ the Boltzmann constant, parameter $\boldsymbol{a}$ is the average distance between regularly arranged pinning centers.

Applying above relations we can predict the dependence of the real critical current, i.e. satisfying the electric field criterion, on the material parameters. Selected results of calculations are presented in Figs. (3-4) and they indicate to the importance of the pinning centers dimensions as well as the elasticity constant $\boldsymbol{\alpha}$ for the critical current magnitude.

From Figs. (3-5) we see that for low pinning centers dimensions as well as for too large elasticity constant of the vortex lattice, the critical current vanishes, which result should have important meaning for technological process. In the case of single crystalline HTc superconductors we should still remember the layered structure of these materials. It concerns especially the $\mathrm{Cu}$-based superconductors although in the recently discovered Fe-based HTc superconductors layered structure appears too. The layered crystal structure of the HTc superconductor $\mathrm{BiSrCaCuO}$ is presented in Fig. (6). 


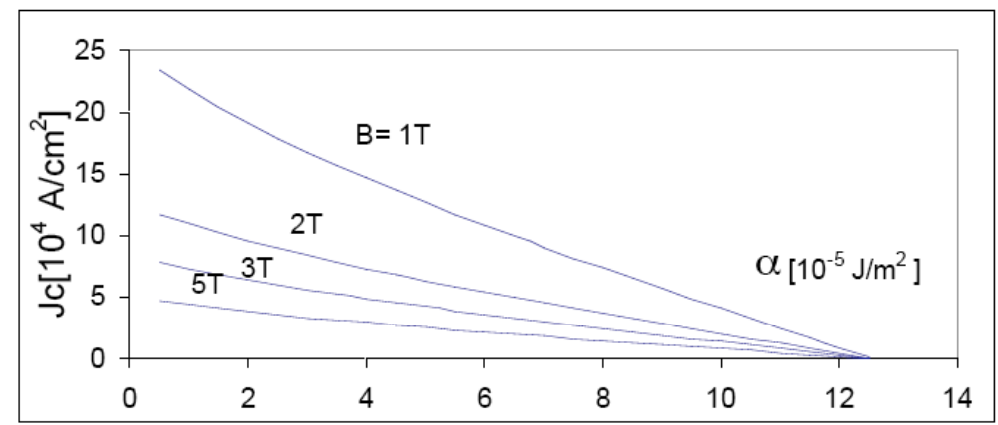

Fig. (5). The dependence of the critical current on the elasticity force of the vortex lattice (described by parameter $\alpha$ ) versus external magnetic induction

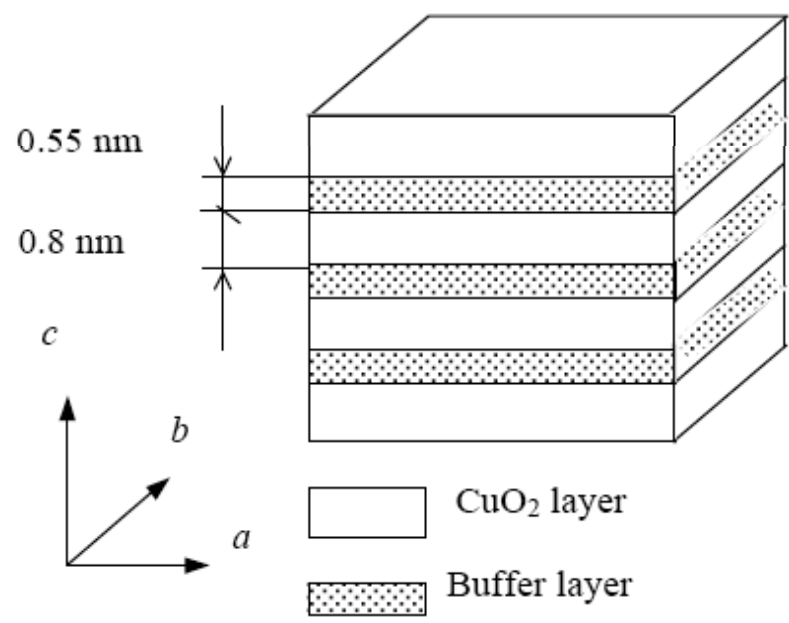

Fig. (6). Scheme of the multilayered structure of the Bi:2212 high temperature superconductor

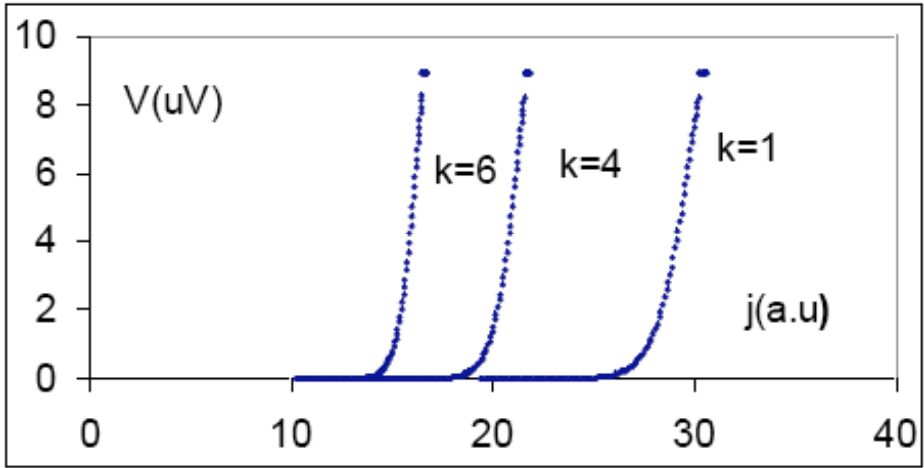

Fig. (7). The influence of the inter-plane interaction $\left(\boldsymbol{k}\right.$ - number of the interacting $\mathrm{CuO}_{2}$ planes) on the current-voltage characteristics of the $\mathrm{HTc}$ layered superconductor

Superconducting layers are intercalated here with the buffer ones, which supply electric charges to the $\mathrm{CuO}_{2}$ layers, responsible for the superconductivity effect, as shown in Fig. (5). The thickness of these layers is smaller than coherence length $\xi_{o}$, so due to the proximity effect the layers interact with neighbouring partners or rather more precisely interact the vortices situated in the neighbouring planes influencing the current-voltage characteristics. The present model allows in the first approximation to consider this interaction, which leads to modification of the current-voltage characteristics in the individual plane, as indicates Fig. (7).

In this Figure it is shown the voltage generated under current flowing in individual plane taking into account the interacting vortices in the neighbouring planes. Index $\boldsymbol{k}$ denotes just the number of interacting planes.

\section{COMPARISON OF THE MODEL OF THE PINNING INTERACTION WITH EXPERIMENTAL DATA}

The above theoretical considerations we have applied then for comparison with experimental data. In Fig. (8) it is shown the comparison of the current-voltage characteristics calculated according to described mathematical model of the pinning interaction with the experimental data obtained for Bi-based superconducting tape. An approximation of the large pinning centers dimensions has been applied here, which means in the language of our model that the case of $\boldsymbol{d}>\boldsymbol{2} \boldsymbol{\xi}$ it has been considered. 


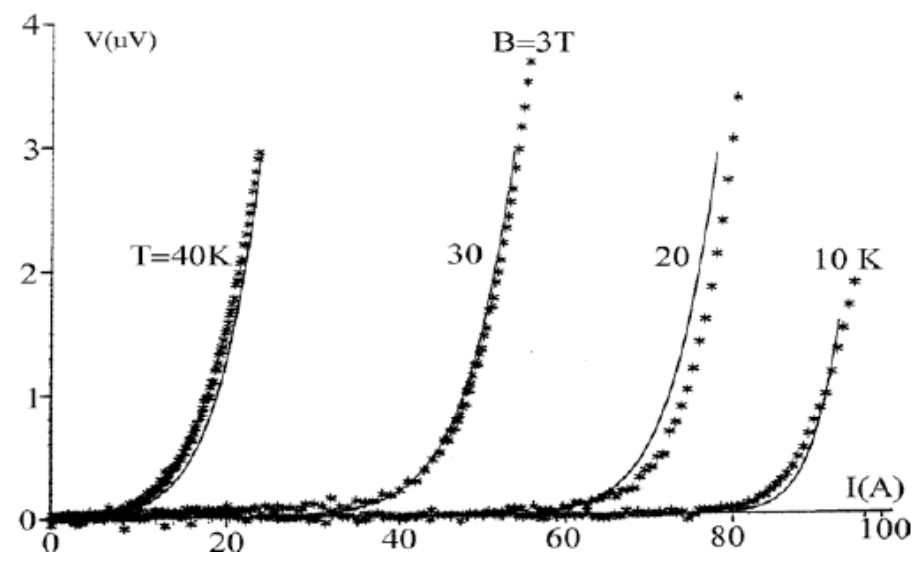

Fig. (8). Comparison of the experimental (*) and theoretical I-V curves for Bi:2223 tape

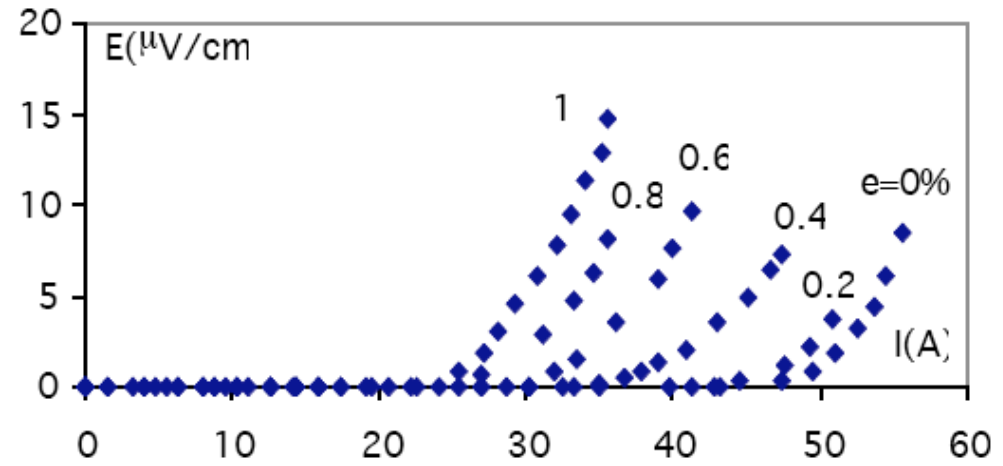

Fig. (9). Experimental dependence of the influence of bending strain on the current-voltage characteristics for the superconducting Bi-2223 tape

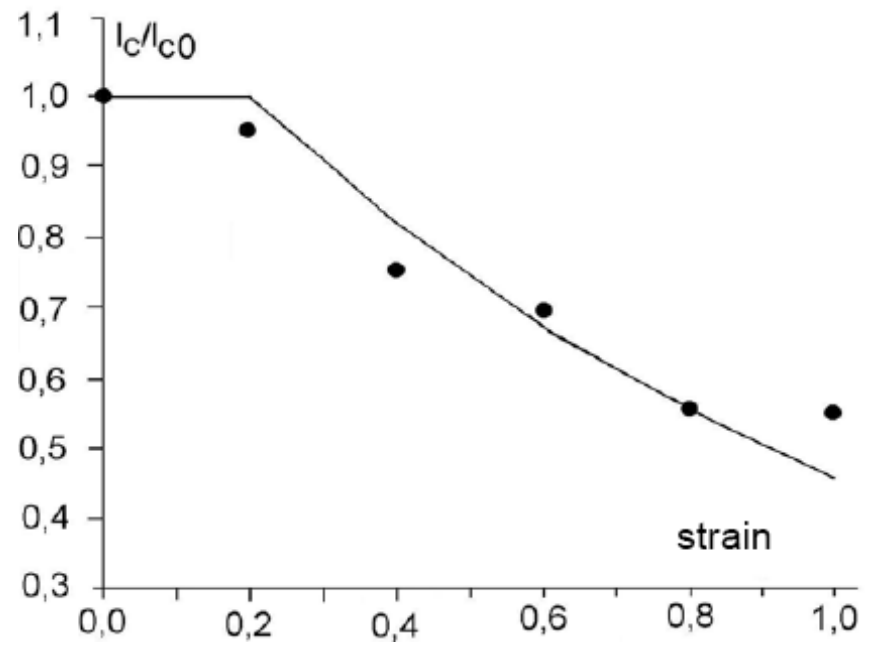

Fig. (10). Experimental dependence of the influence of bending strain on the critical current of the superconducting Bi-2223 tape

Very good agreement of the theoretical model with the experimental data has been received at various temperatures, while using common fitting parameter, which is the concentration of the pinning centers. It should be noticed that the defects concentration received from the scanning electron microscopy analysis indicated to similar value.

Additionally, structural defects such as micro-cracks are created as the result of the bending strain of the superconducting tapes, which effect frequently appears during winding of the superconducting coils. Experimental data of the influence bending strain on the current-voltage characteris- tics are given in Fig. (9), while critical current data versus bending strain is shown in Fig (10). Bending strain parameter $\boldsymbol{e}$, which appears in the Figs. (9-10) is defined as the ratio of the tape thickness $t$ to its diameter $D: e=t / D$.

In Fig. (11) is presented the theoretical effect of the bending strain on the current-voltage characteristics, calculated by taking into account the present form of the pinning interaction.

These theoretical results are in qualitative agreement with the experimental data shown in Fig. (9). Knowledge of the 


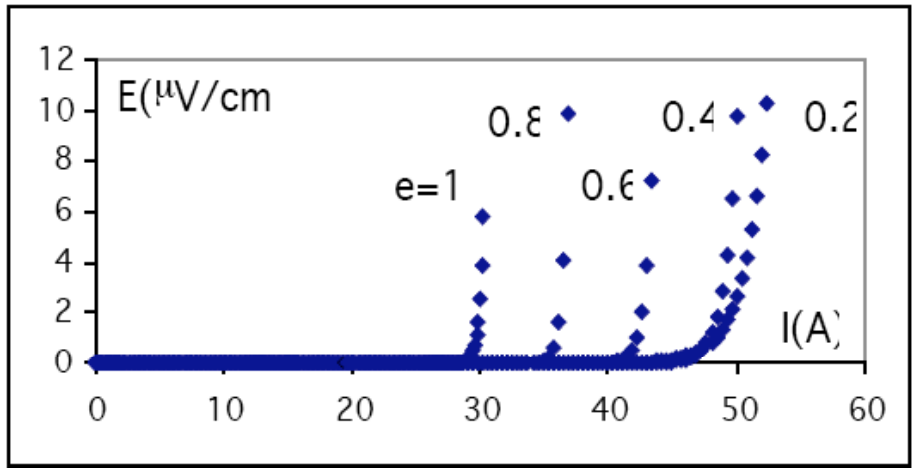

Fig. (11). Theoretically predicted influence of the bending strain $\boldsymbol{e}$ (in percents), on the current-voltage characteristics for Bi:2223 tape

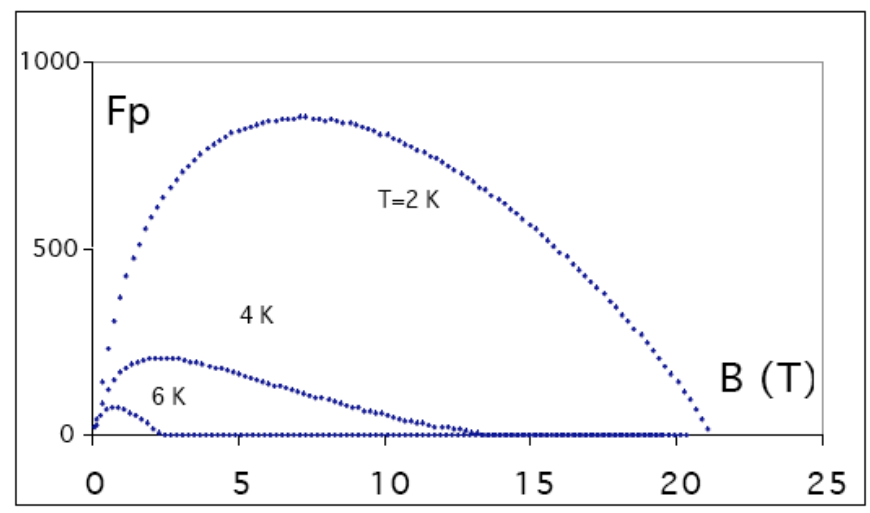

Fig. (12). Theoretical dependence of the flux pinning on the magnetic induction versus temperature

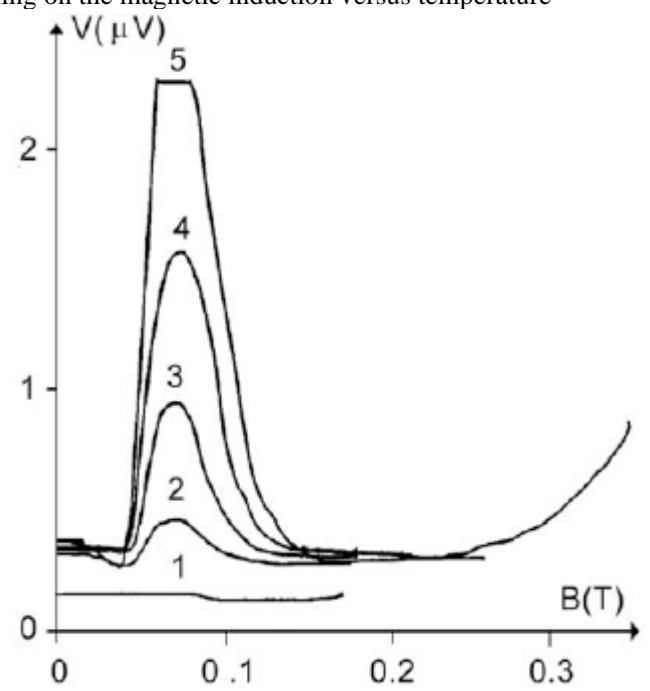

Fig. (13). Experimental dynamical anomalies of the current-voltage characteristics of $\mathrm{YBaCuO}$ ceramic, in function of the current amplitude. For curves 1-5 current is varying between 0 and $200 \mathrm{~mA}$ respectively [11]

critical current allows us to determine the volume pinning force, which is force acting on vortices in the unit volume. To determine this force there should be taken into account many body problem of the occupation varying with magnetic field number of the vortices on the fixed number of the pinning centers. Final result of the theoretical magnetic field dependence of the pinning force for three temperatures is shown in Fig. (12). The shape of this curve is in agreement with experimental data [10] measured on Bi-2223 tapes.

Theoretical model, which results are shown in Fig. (12), predicts the shift of the maximal value of the pinning force with temperature, which was observed experimentally in [10]. The presented model allows to explain also the dynamic anomalies of the current-voltage characteristics of the HTc ceramics observed by us previously [11] and shown in Fig. (13).

\section{INFLUENCE OF THE INITIAL CAPTURED VOR- TEX POSITION ON THE CRITICAL CURRENT DENSITY}

In detailed analysis of the pinning interaction we should still consider the mechanism of vortex capturing on the pinning centers. Two topics are therefore regarded now, con- 
nected with the geometrical aspects of this capturing process. The first one is related to the initial position of the captured vortex, while the second to the renormalization of the screening currents around the vortex captured on the nano-sized pinning center. Now we consider this first point, while the second one will be the subject of the next clause 5. In the previous point it has been considered the vortex dynamics problem for the initial state of the vortex captured onto the depth of the renormalized coherence length, as shows Fig. (2). Such configuration is preferred because it should allow among other still for the flow of the superconducting screening currents, around the vortex core, due to the proximity effect and keep this way the vortex structure. For evaluating the relevance of this initial configuration for the final results describing the potential barrier height and critical current density, the independent calculations have been performed for another initial vortex state. We have considered therefore additionally the configuration of the fully captured vortex, it is the vortex core pinned at the depth of $2 \xi$ inside the pinning center of the nanometric size. The basic equations describing the initial state normal energy for the fully captured vortex as well as for the vortex shifted against this initial position have been derived according to the method presented in the clause 2 , while the results are given below. Initial normal state energy of the fully captured vortex, according to the notation of the Fig. (2) is equal to:

$$
U(-\xi)=\frac{\mu_{0} H_{c}^{2} l_{p}}{2}\left[\pi \xi^{2}-2 \xi^{2} \arcsin \frac{d}{2 \xi}-d \sqrt{\xi^{2}-\frac{d^{2}}{4}}\right]
$$

For the first deflection of the vortex from this initial position it appears the dependence:

$$
U_{1}(x)=\frac{\mu_{0} H_{c}^{2} l_{p}}{2}\left[\begin{array}{l}
\pi \xi^{2}-2 \xi^{2} \arcsin \frac{d}{2 \xi}+ \\
\xi^{2} \arcsin \frac{\sqrt{\xi^{2}-x^{2}}}{\xi}-d \sqrt{\xi^{2}-\frac{d^{2}}{4}} \\
+x \sqrt{\xi^{2}-x^{2}}
\end{array}\right]
$$

valid for the following range of the vortex deflection:

$$
-\xi \leq x \leq-\xi \sqrt{1-\left(\frac{d}{2 \xi}\right)^{2}}
$$

For the next range of the vortex movement described by the new boundary condition:

$$
-\xi \sqrt{1-\left(\frac{d}{2 \xi}\right)^{2}} \leq x \leq \xi \sqrt{1-\left(\frac{d}{2 \xi}\right)^{2}}
$$

the normal state energy of the shifted vortex is given by the relation:

$$
U_{2}(x)=\frac{\mu_{0} H_{c}^{2} l_{p} \xi^{2}}{2}\left[\pi-\arcsin \frac{d}{2 \xi}-\frac{d}{2 \xi} \sqrt{1-\left(\frac{d}{2 \xi}\right)^{2}}+d x\right]
$$

For still larger vortex shift the normal state potential is:

$$
U_{3}(x)=\frac{\mu_{0} H_{c}^{2} l_{p} \xi^{2}}{2}\left[\frac{\pi}{2}+\arcsin \frac{x}{\xi}+\frac{x}{\xi} \sqrt{1-\left(\frac{x}{\xi}\right)^{2}}\right]
$$

Expressions for the potential barrier height, which determine the current-voltage characteristics have been derived then and are given below for each of the considered cases:

$$
\Delta U_{1}(x)=\frac{\mu_{0} H_{c}^{2} l_{p}}{2}\left[\xi^{2} \arcsin \frac{\sqrt{\xi^{2}-x^{2}}}{\xi}+x \sqrt{\xi^{2}-x^{2}}\right]
$$

for the values of the vortex shift described by parameter $\mathrm{x}$, in the range given by Eq. 14 .

For larger shifts of the vortex in the range defined by Eq. 15 , the potential barrier height is equal to:

$$
\Delta U_{2}(x)=\frac{\mu_{0} H_{c}^{2} l_{p}}{2}\left[\xi^{2} \arcsin \frac{d}{2 \xi}+\frac{d}{2 \xi} \sqrt{\xi^{2}-\frac{d^{2}}{4}}+d x\right]
$$

For still higher vortex deflection potential barrier height is described by the relation:

$$
\Delta U_{3}(x)=\frac{\mu_{0} H_{c}^{2} l_{p}}{2}\left[\begin{array}{l}
-\frac{\pi \xi^{2}}{2}+\xi^{2} \arcsin \frac{x}{\xi}+ \\
x \xi \sqrt{1-\left(\frac{x}{\xi}\right)^{2}}+2 \xi^{2} \arcsin \frac{d}{2 \xi} \\
+d \sqrt{\xi^{2}-\left(\frac{d}{2}\right)^{2}}
\end{array}\right]
$$

Transforming the above dependences 18 - 20 from translational into the current representation, in the case of the full vortex capturing we obtain final relation describing the potential barrier height in the function of the current density $\boldsymbol{i}=$ $j / j_{c}$, reduced to the critical one:

$$
\Delta U_{3}(i)=\frac{\mu_{0} H_{c}^{2} l_{p} \xi^{2}}{2}\left[\begin{array}{l}
-\arcsin (i)-i\left(2+\sqrt{1-i^{2}}\right)+ \\
2 \arcsin \frac{d}{2 \xi}+\frac{d}{\xi} \sqrt{1-\left(\frac{d}{2 \xi}\right)^{2}}
\end{array}\right]
$$

Eq. 21 we insert then into the constitutive equation 9 describing the current-voltage characteristics in the function of the potential barrier height $\boldsymbol{\Delta U}$. The results of the comparison of the calculations of the current-voltage characteristics for both (fully captured and half captured) initial states are shown in Fig. (14). For better recognition of the behavior of the considered model describing current-voltage characteristics of HTc superconductors, the calculations have been performed as previously for various magnetic fields. As it follows from Fig. (14) for both cases it is for the fully captured vortex as well as for the half-captured the differences in the current - voltage characteristics are at least in this case negligible.

The influence of an initial position of the captured vortex on the critical current of the HTc superconductor in reduced units versus pinning center width is presented in Fig. (15). 


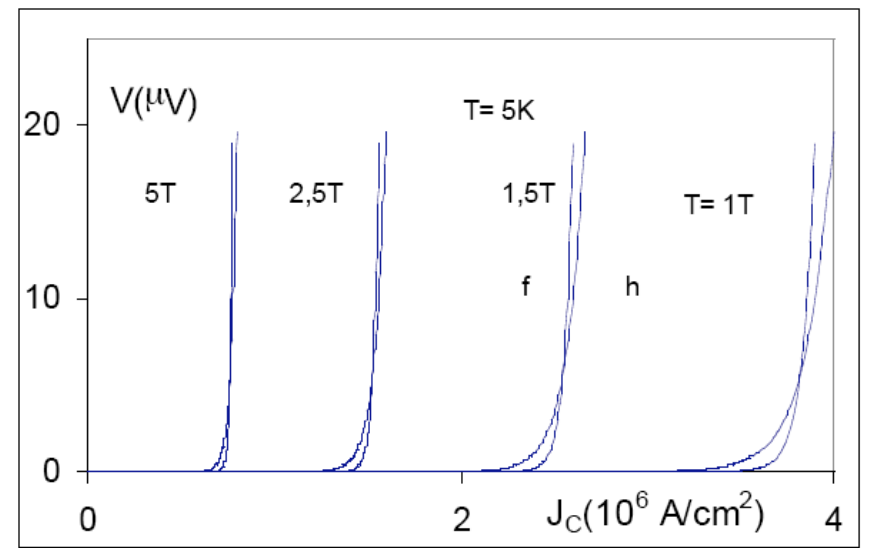

Fig. (14). The current-voltage characteristics of the HTc superconductor with half pinned vortex (h) in initial state and fully pinned (f) for d/2 $\xi=0.4$ versus the applied external magnetic field.

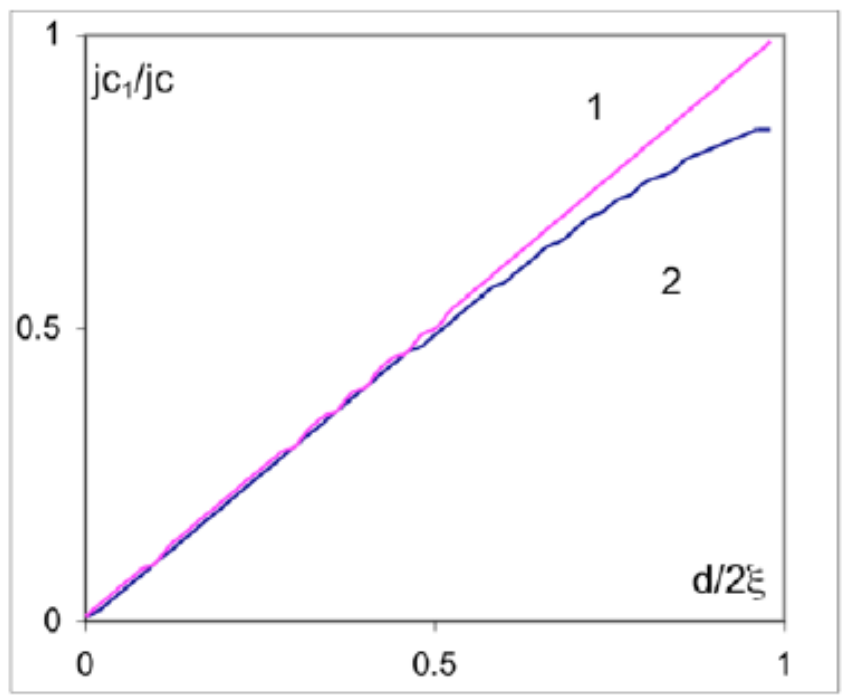

Fig. (15). The dependence of the reduced critical current density on the pinning center width, for two initial positions of the vortex: 1 - half captured, 2 - fully captured.

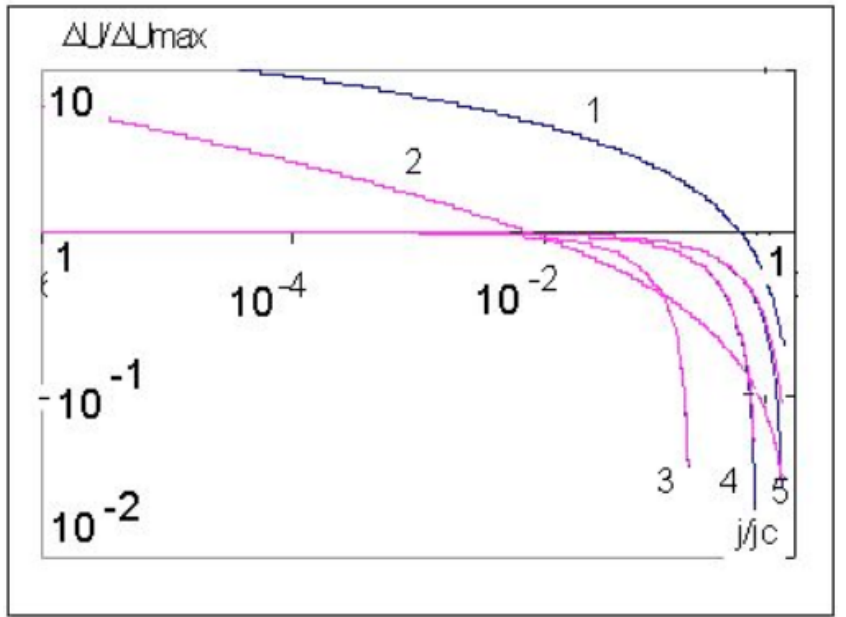

Fig. (16). The dependence of the reduced potential barrier on the current normalized to the critical one for: (1) logarithmic dependence ln $\left(\mathrm{j} / \mathrm{j}_{\mathrm{c}}\right)$ of the potential barrier, (2) dependence of the power like form $\left(\mathrm{j} / \mathrm{j}_{\mathrm{c}}\right)^{-1 / 7}-1$, (3) present model for $\mathrm{d} / 2 \xi=0,15$, (4) present model for $\mathrm{d} / 2 \xi=0,5(5)$ present model for $\mathrm{d} / 2 \xi=0,95$.

The critical current density $j_{c l}$ shown in this figure is defined as the current density leading to the disappearance of the energy barrier $\Delta U$ in the given case, while $j_{c}$ has been defined previously in Eq. 5.
Fig. (16) on the other hand shows the comparison of the reduced potential barrier versus the current normalized to the critical one for various pinning forces models [12-13], bringing logarithmic and power-like dependence of the potential 

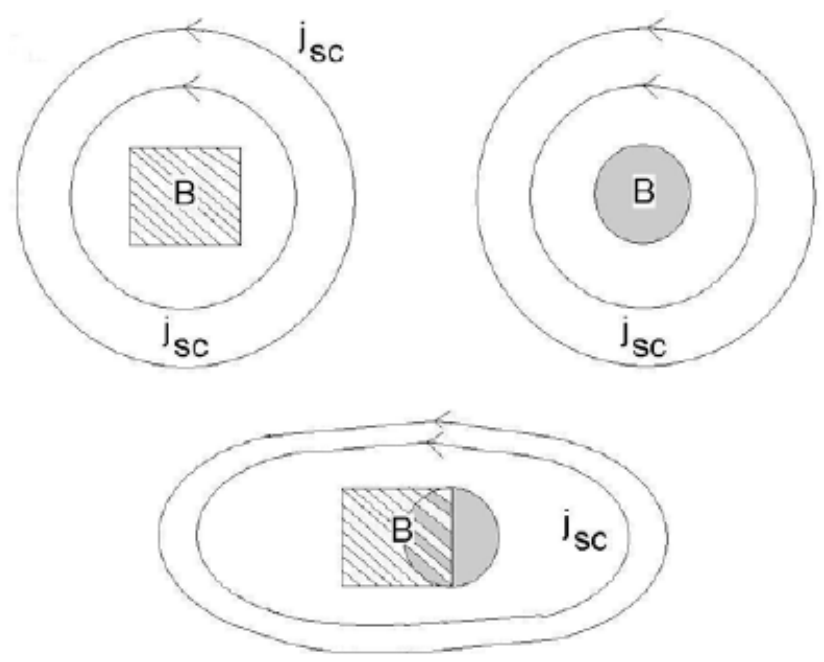

Fig. (17). Schematic comparison of the screening currents distribution and magnetic induction penetration into the vortex core and isolated nano-sized defect (at the top) and for the captured vortex (at the bottom) in HTc superconductor.

barrier. The results obtained in the present approach are marked by numbers 3-5 here and are similar in fact, for both limiting cases, of the full and half captured vortices in the initial state.

\section{INFLUENCE ON THE CRITICAL CURRENT OF THE MODIFICATION OF THE SCREENING CURRENT DISTRIBUTION IN THE CAPTURED VORTEX}

In previous points we have considered the model describing the pinning interaction based on the energy balance, in which there has been applied an assumption of an unchanged electromagnetic shape of an isolated, free vortex and for the vortex captured on the nano-sized pinning center. In fact however we should remember that the vortex is composed from the circulating currents around the vortex core. In a captured vortex the screening current distribution is modified as it indicated schematically in Fig. (17).

Generally description of such configuration is very complicated from the mathematical point of view. In the present paper we have applied therefore a very simplified approach, in which has been considered according to Fig. (17) the generalized magnetic flux quantization. For an isolated vortex and nano-sized pinning center the flux quantization conditions are fulfilled separately for both objects. However for the captured vortex the screening currents distribution is renormalized, while the quantization condition concerns now the total object of the captured vortex and pinning center.

This very complicated effect in the first approximation we have described by an assumption of the modification of the induction distribution in the vortex core of the captured vortex. We have considered therefore the case in which magnetic field penetrates the superconducting material not only through the cores of the magnetic vortices but also by the normal state inclusions, observed just in the scanning electron microscopy, for instance holes and normal grains. The Fig. (18) presents above geometry. It shows the pancake vortex captured on the nano-pinning center of rectangular shape. If a vortex leaves the pinning center then the flux in- side it is quantized and brings the value of $\Phi_{0}=2,067 \cdot 10^{-15}$ $\mathrm{Wb}$.

For captured vortex magnetic flux penetrating its core should be treated as the superposition of the magnetic field passing the normal state inclusion, which is proportional to the external magnetic field applied and the quantized flux of the magnetic vortex. It is generally a very complicated effect, connected with the variation of the current distribution, because screening currents now surround both the vortex core as well as the nano-sized defect. We notice also that there appear sometimes suggestions of transporting by the vortex not only individual flux quantum but also its multiplication, despite of non - favours energetically such configuration, whose ideas are partly similar to presented in this approach.

In this first approach we apply here a rather rough approximation of an unchanged cylindrical symmetry of the vortex structure even in the captured state. If we approximate further the magnetic induction profile in the vortex, through the London's relation:

$$
B(r)=B(0) \exp \left(-\frac{r}{\lambda}\right)
$$

then the flux quantization condition leads to the following integral:

$\Phi_{0}=2 \pi \int_{0}^{\infty} B(0) \exp \left(-\frac{r}{\lambda}\right) r d r=2 \pi \lambda^{2} B(0)$

$\boldsymbol{B}(\boldsymbol{\theta})$ is the magnetic induction in the middle of the vortex core, which now is simply proportional to the total magnetic flux in the vortex:

$$
B(0)=\frac{\Phi_{0}}{2 \pi \lambda^{2}}
$$

Eq. 24 indicates clearly that in the first approximation above the magnetic flux carried by the vortex determines the magnetic induction in the center of the vortex. It is especially important for the pinned vortex, whose geometry is pre- 


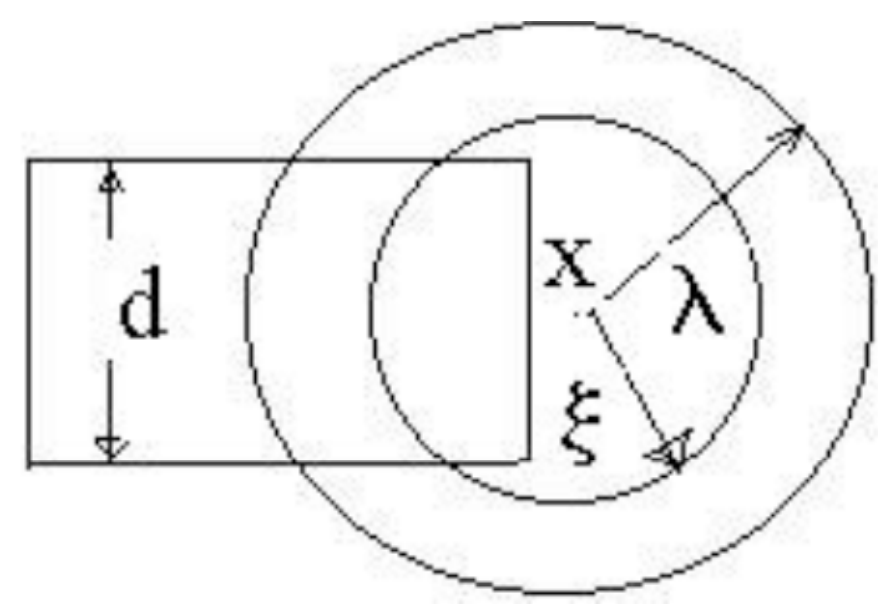

Fig. (18). View of the vortex captured on the nano-sized pinning center. The vortex core radius is equal to the coherence length $\xi$, while full vortex size is of the range of the penetration depth $\lambda$.

sented in the Fig. (18). The flux passing through the captured vortex area, is (according to the present, simplified model) significantly modified in the respect to the conditions 23-24. Thus magnetic flux equal to $\Phi_{0}$ is quantized in isolated vortex, while in captured one, due to the significant modification of the electromagnetic circuit quantized should be already the total flux passing through the vortex core and the magnetic flux crossing the pinning center. In the first approximation we have assumed therefore, according to these assumptions and scheme shown in Fig. (18), that the magnetic flux in the region of the captured vortex is enhanced in comparison to an isolated one. The magnetic flux in the captured vortex, is then a superposition of the flux quantum and of the magnetic field appearing in the common region of the pinned vortex and empty hole or normal metal inclusion, acting as the pinning center. As it was stated previously, we assume further additionally that the cylindrical geometry of the pinned vortex remains unchanged. In the initial state of the pancake vortex captured on the pinning center, as it shows Fig. (18), the enhanced magnetic flux $\Phi_{l}$ in the vortex area is therefore according to these considerations equal:

$$
\frac{\Phi_{1}}{\Phi_{0}}=1+\frac{B_{e} \lambda^{2}}{\Phi_{0}}\left[\arcsin \frac{d}{2 \lambda}+\frac{d}{2 \lambda} \sqrt{1-\left(\frac{d}{2 \lambda}\right)^{2}}\right]
$$

where $\boldsymbol{B}_{\boldsymbol{e}}$ is the magnetic induction, passing through the pinning center, while $\boldsymbol{d}$ denotes the width of the pinning center of a rectangular shape. Parameter $\boldsymbol{B}_{\boldsymbol{e}}$ is roughly proportional to the applied external magnetic induction. For the case of the vortex slightly shifted from the pinning center the magnetic flux inside it is given by the following formula:

$$
\frac{\Phi_{1}}{\Phi_{0}}=1+\frac{B_{e} \lambda^{2}}{\Phi_{0}}\left[\begin{array}{l}
\arcsin \frac{d}{2 \lambda}+\frac{d}{2 \lambda} \sqrt{1-\left(\frac{d}{2 \lambda}\right)^{2}} \\
-\frac{d}{\lambda} \cdot \frac{x}{\lambda}
\end{array}\right]
$$

while for deflection of the vortex core from the pinning center larger than $\boldsymbol{x}_{\boldsymbol{c} 1}$ the magnetic flux in the vortex area is equal to:

$$
\frac{\Phi_{1}}{\Phi_{0}}=1+\frac{B_{e} \lambda^{2}}{\Phi_{0}}\left[\frac{\pi}{2}-\arcsin \frac{x}{\lambda}-\frac{x}{\lambda} \sqrt{1-\left(\frac{x}{\lambda}\right)^{2}}\right]
$$

Parameter $\boldsymbol{x}_{\boldsymbol{c} l}$ denotes the limiting value of the vortex deflection distinguishing these both regions, for which Eq. 24 or Eq. 25 are valid respectively and is described by the relation:

$$
x_{c 1}=\lambda \sqrt{1-\left(\frac{d}{2 \lambda}\right)^{2}}
$$

As it follows from Eq. 27, for fully released vortex, it is shifted on the length $\mathrm{x}=\lambda$, the magnetic flux in vortex reaches the magnitude of $\Phi_{0}$. The magnetic flux $\Phi_{1}$ penetrating vortex determines then according to Eqs. 23-24 the maximum magnetic induction in the center of vortex core $\boldsymbol{B}(\boldsymbol{\theta})$ and influences in this way magnetic induction distribution inside the vortex, according to the relation 22. Conditions 22-24 determine the new value of the vortex core radius, which creates normal state part of the vortex, in the language of Fig. (18) described by the effective coherence length, may be slightly different from original coherence length. The new radius of the vortex core $\xi_{l}$ is determined according to Eqs. $25-27$ by the relation:

$$
\mu_{0} H_{c}=\frac{\Phi_{1}}{2 \pi \lambda^{2}} \exp \left(-\frac{\xi_{1}}{\lambda}\right)
$$

where $\boldsymbol{H}_{\boldsymbol{C}}$ is thermodynamic critical magnetic field, $\mu_{0}$ magnetic permeability, while magnetic flux $\Phi_{1}$ is described by Eqs. 25-27. We apply then the condition joining the thermodynamic critical magnetic field with coherence length of an isolated vortex:

$$
\mu_{0} H_{c}=\frac{\Phi_{0}}{2 \pi \lambda^{2}} \exp \left(-\frac{\xi_{0}}{\lambda}\right)
$$

where $\boldsymbol{\Phi}_{\boldsymbol{0}}$ and $\boldsymbol{\xi}_{\boldsymbol{0}}$ describe the flux quantum and normal core radius of an isolated vortex. According to Eqs. 29 - 30 the expression for the modified length of the normal core radius is given by the relation: 


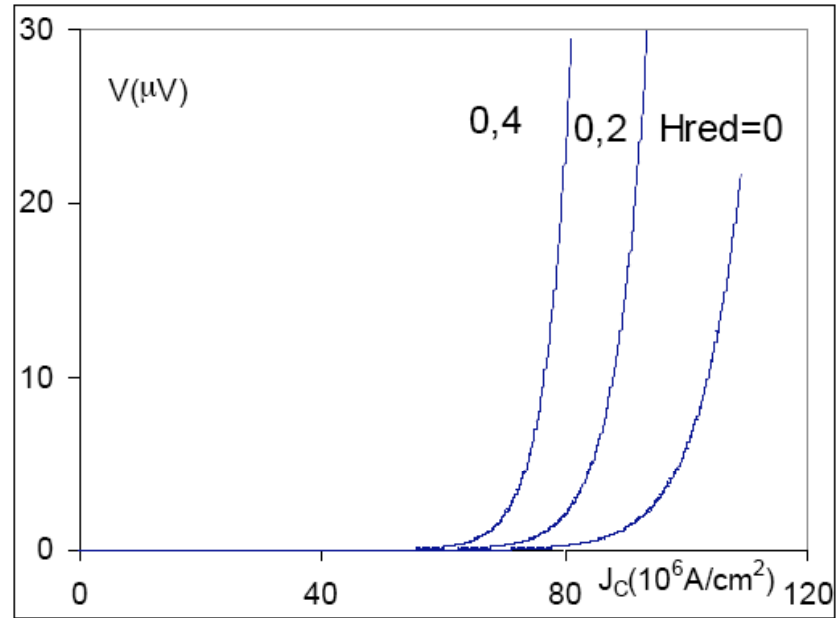

Fig. (19). Calculated current-voltage characteristics for the HTc superconductor as a function of the reduced applied magnetic field $H_{\text {red }}$

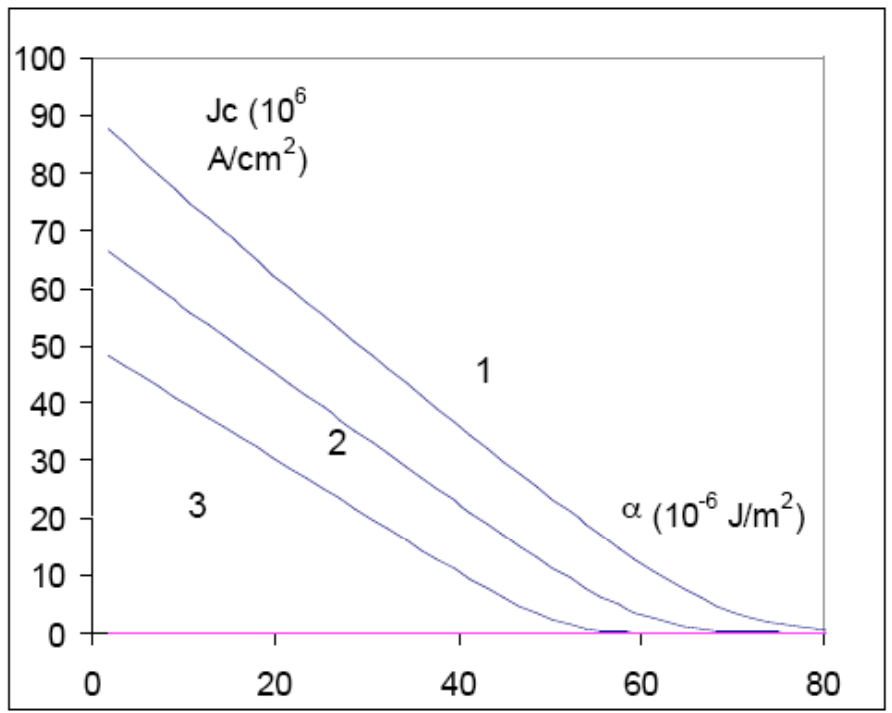

Fig. (20). The dependence of the critical current density on the rigidity of the vortex lattice, expressed by the parameter $\boldsymbol{\alpha}$ as a function of the magnetic flux $\Phi_{1}:$ (1) $\Phi_{1}=\Phi_{0}(2) \Phi_{1}>\Phi_{0}(3) \Phi_{2}>\Phi_{1}$

$$
\frac{\xi_{1}}{\xi_{0}}=1+\kappa \ln \frac{\Phi_{1}}{\Phi_{0}}
$$

where the Ginzburg-Landau theory parameter $\kappa=\frac{\lambda}{\xi_{0}}$ characterizes the kind of the superconductor. The present approach taking into account in the first approximation the electromagnetic nature of the interaction pancake vortex with the nano-sized pinning centers, leads therefore to the modification of the normal state core radius $\xi_{1}$ of the captured vortex. This parameter has essential meaning for an analysis of the energy barrier in the flux creep process, as it was shown in point 2.

The results of the calculations current-voltage characteristics according to elaborated model in the function of the reduced magnetic field $H_{r e d}=\frac{B_{e} \lambda^{2}}{\Phi_{0}}$ are shown in Fig (19).

The strong influence of the value $\boldsymbol{H}_{\text {red }}$ on the current-voltage characteristics is well seen in Fig. (19). The above result is in agreement with an experimental observation, that the applied magnetic field reduces the critical current. The critical current density is determined then, as the value for which potential barrier vanishes. The influence on the critical current density of the parameter $\alpha$, describing the rigidity of the vortex lattice, also as a function of the magnetic flux $\Phi_{1}$ appearing in the relations 25-27 is presented in Fig (20).

The new model of the pinning mechanism allows therefore to predict the critical current of the HTc materials as a function of the vortex capturing parameters. The critical current is an essential parameter from the point of view of application of HTc materials in the energy transport process. On the other hand critical current determines also the screening properties of bulk HTc superconductors, which are important for application of these materials as permanent magnet, for instance in levitation processes. We should notice that in bulk HTc materials, due to their granular structure, there appear not only intragranular currents but also intergarnular, which can be treated as Josephson's like currents. The peculiar screening properties of HTc superconductors 


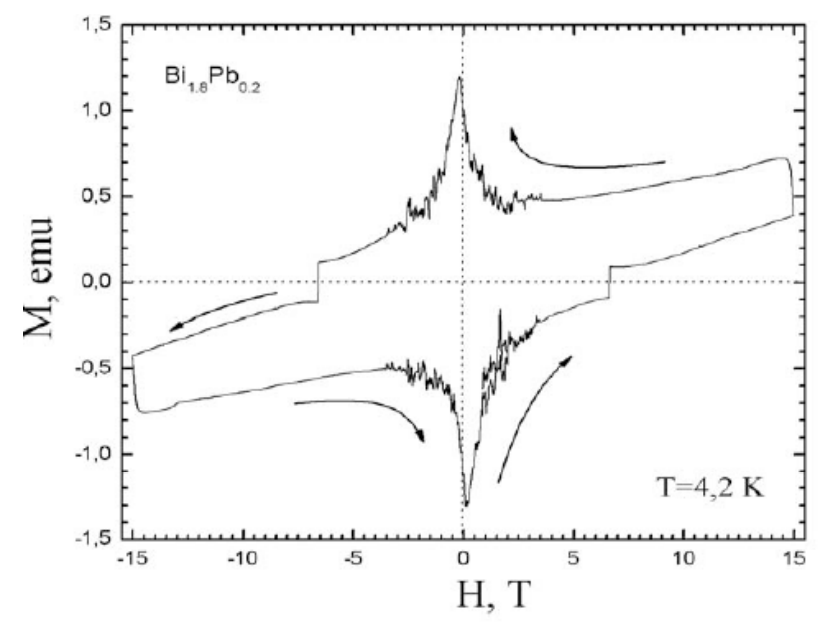

Fig. (21). Full magnetic hysteresis loop of the $\mathrm{Bi}_{1.8} \mathrm{~Pb}_{0.2} \mathrm{Sr}_{2} \mathrm{Ca}_{2} \mathrm{Cu}_{3} \mathrm{O}_{8} \mathrm{HTc}$ superconducting ceramic in high magnetic field

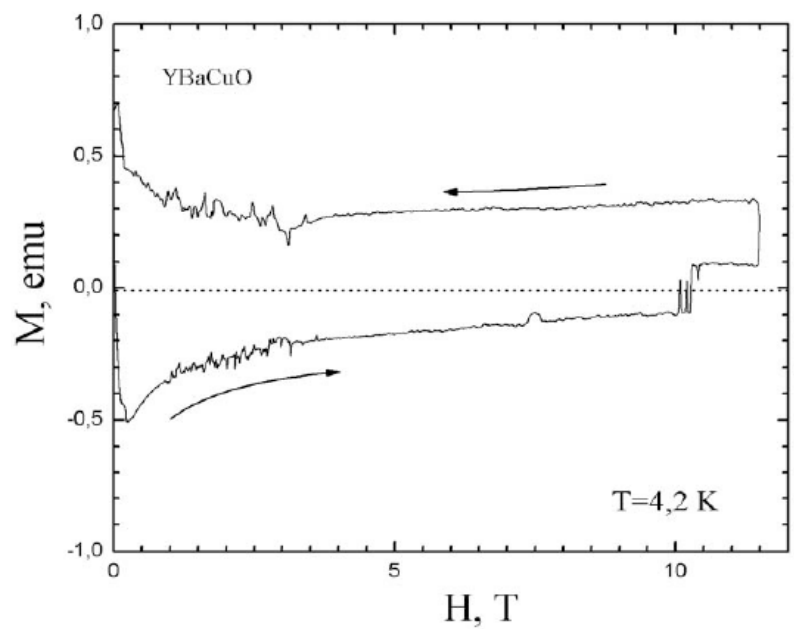

Fig. (22). Full magnetic hysteresis loop of the $\mathrm{YBa}_{2} \mathrm{Cu}_{3} \mathrm{O}_{7-\mathrm{x}} \mathrm{HTc}$ superconducting ceramic in high magnetic field.

are related to the flux trapping, while analysis of this effect is performed in the next point.

\section{TRAPPED MAGNETIC FLUX ANALYSIS IN HTC CERAMICS}

The trapped magnetic flux (Ftr) is an important parameter dependent on the flux pinning and critical current. It is defined as the remanent moment of the magnetization curve in the magnetic field cycle $0 \rightarrow B_{m} \rightarrow 0$, where $B_{m}$ is the maximal magnetic induction in the cycle. An example of irreversible magnetization curves for a high temperature superconductor (Bi-2223 bulk ceramic) and $\mathrm{YBa}_{2} \mathrm{Cu}_{3} \mathrm{O}_{7-\mathrm{x}}$ ceramic superconductor is shown in Figs. (21-22). Both these curves indicate to the existence of magnetic irreversibility, which means that trapped flux is common property of type II superconductors. The existence of small flux jumps is also observed here for both directions of the magnetic field variation.

The trapped magnetic flux is measured by the Hall probe method as well as just by the remanent moment of the magnetization curve. In best macro-granular $\mathrm{YBaCuO}$ samples the trapped flux reaches $17 \mathrm{~T}$ at $29 \mathrm{~K}$, which value clearly indicates to the magnitude of this effect in HTc superconductors and creates therefore the attractive possibility of applying these materials as the permanent magnets. Fig. (23) pre- sents the calculated model of a levitating train using permanent magnets oppositely oriented and levitating above them a HTc superconductor in Meissner state. Alternative experiments are performed for the superconductor in the flux trapped state suspended below shown in Fig. (23) permanent magnets.

Fig. (24). shows magnetic field profiles in the superconducting bearing constructed from HTc superconducting tube rotating on a core composed from six permanent magnets.

Fig. (25) presents the scheme of the magnetic induction distribution in the flux trapped state. Existence of the intergranular Josephson's currents, mentioned previously and the current inside grains lead to the tooth-like shape of the magnetic induction profile given schematically here.

In order to analyse the trapped flux value we have considered four cases dependent on the amplitude of the external magnetic induction $\boldsymbol{B}_{\boldsymbol{m}}$ in the magnetizing cycle. The first case finds place for the maximum induction $\boldsymbol{B}_{\boldsymbol{m}}$ lower than the first penetration field $\boldsymbol{B}_{\boldsymbol{c l}}$. Then the magnetic induction does not penetrate into the volume of the superconductor, while the trapped flux $F_{\text {tr }}$ vanishes therefore. In the second region of magnetic field variations described by the relation: $\xi>B_{m}^{1}>0$, where $\boldsymbol{B}_{\boldsymbol{m}}{ }^{1}=\boldsymbol{B}_{\boldsymbol{m}}-\boldsymbol{B}_{\boldsymbol{c} 1}$, trapped flux being mathe- 

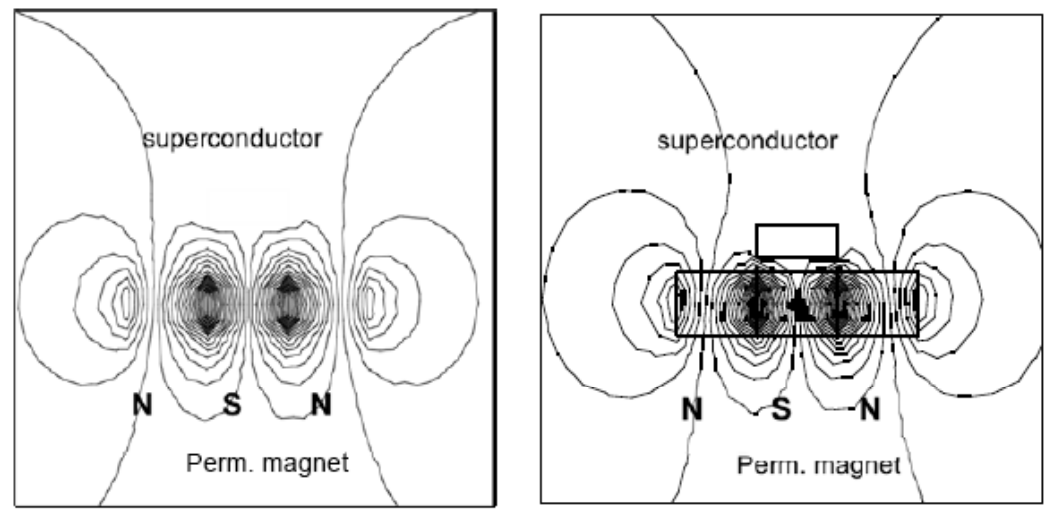

Fig. (23). Calculated magnetic field lines in the model of the levitating train - superconductor above two oppositely oriented permanent magnets, for two distances

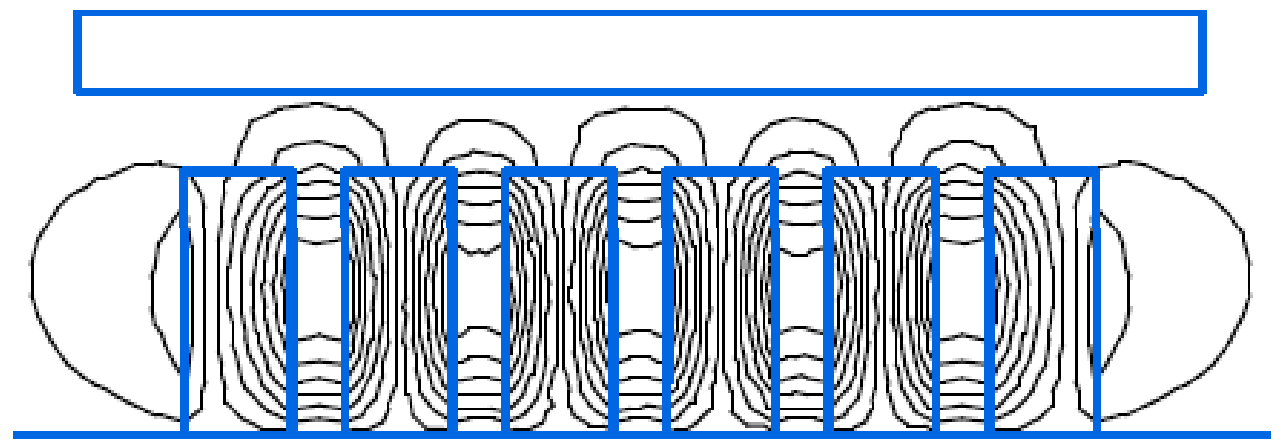

Fig. (24). Cross-section of the magnetic bearing composed from the HTc superconducting cylinder levitating above 6 permanent magnets.

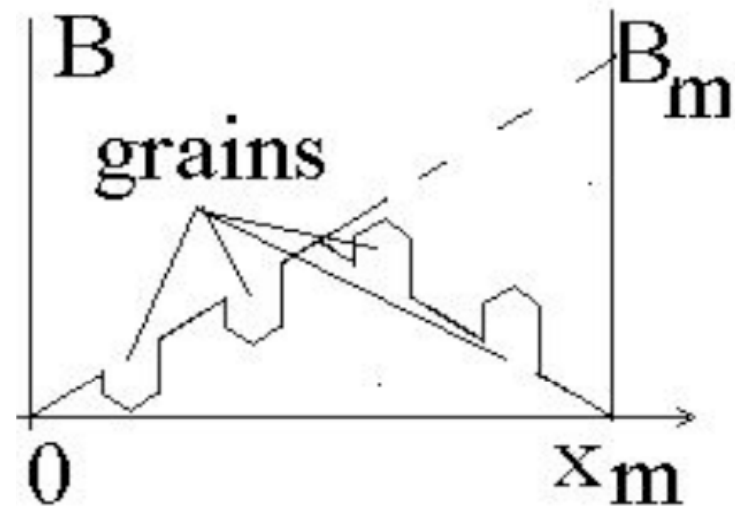

Fig. (25). Magnetic induction profile in the flux trapped state of the HTc ceramic superconductor for the magnetic field cycle $0 \rightarrow B_{m} \rightarrow 0$. Sharp changes of the magnetic distribution are due to existence of the grains.

matically the integral over the superconductor volume from the induction profile shown in the Fig. (25) is expressed by the following relation, in an approximation of the constant current density in the cylindrical geometry sample:

$$
F_{t r}=\frac{B_{m}^{1^{2}}}{2 \xi^{2}}\left[\xi-\frac{B_{m}^{1}}{2}+n B_{s g}\right]
$$

Parameter $\boldsymbol{\xi}$ describes here the full penetration magnetic induction inside the superconducting material of the radius $\boldsymbol{R}$, without surface barrier effects:

$$
\xi=\mu_{0} j_{c} R
$$

In Eq. 32 has been taken into account according to the Fig. (25) the existence of the superconducting grains immersed into intergranular matrix, filling of which is described by the relative concentration $\boldsymbol{n}$, varying between 0 and 1. An amount of the trapped flux connected with an individual grain, divided on its cross-section we denote by the symbol $\boldsymbol{B}_{s g}$, described by the dependence:

$B_{s g}=B c_{1 g}+\frac{\xi g}{3}$

$\boldsymbol{B} \boldsymbol{c}_{1 g}$ denotes the first critical magnetic field in the superconducting grains, while $\xi_{g}$ is determined by the relation:

$\xi g=\mu_{0} j_{c g} R_{g}$ 


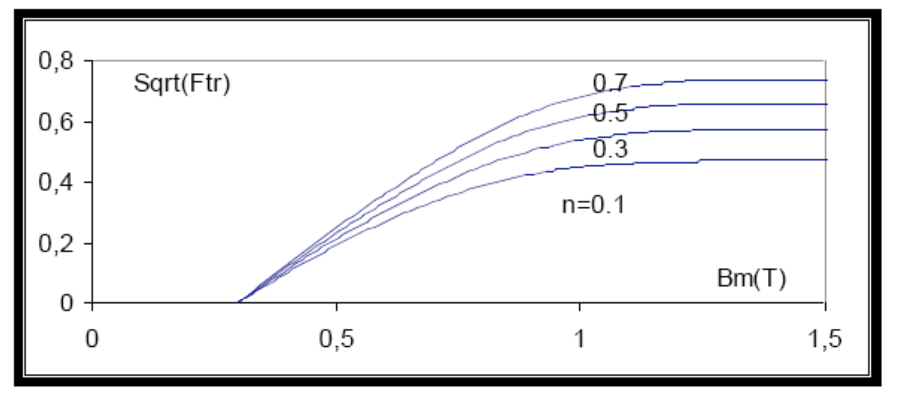

Fig. (26). The influence of the filling with superconducting grains of the superconductor on the square root of the flux trapping Ftr. Numbers at curves indicate to values of the reduced grain concentration varying between $0<n<1$.

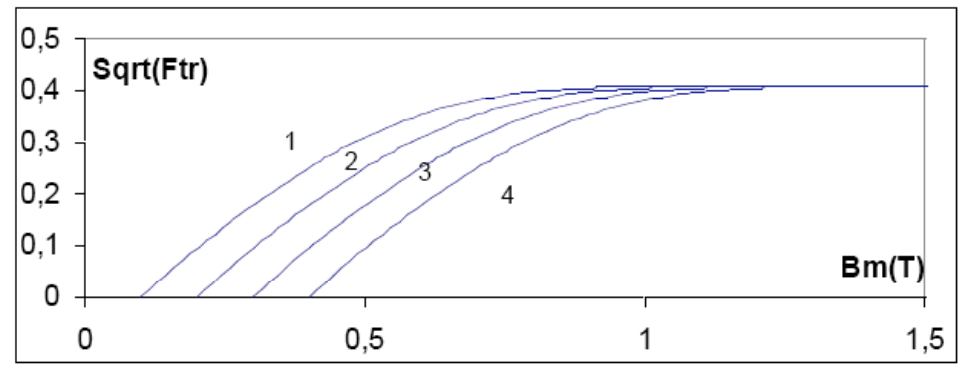

Fig. (27). The influence of the first critical magnetic field $\boldsymbol{B}_{c 1}$ of the superconducting matrix on the trapped magnetic flux Ftr: (1) $\mathrm{B}_{\mathrm{c} 1}=100 \mathrm{mT}$, (2) $200 \mathrm{mT}$, (3) $300 \mathrm{mT}$, (4) $400 \mathrm{mT}$.

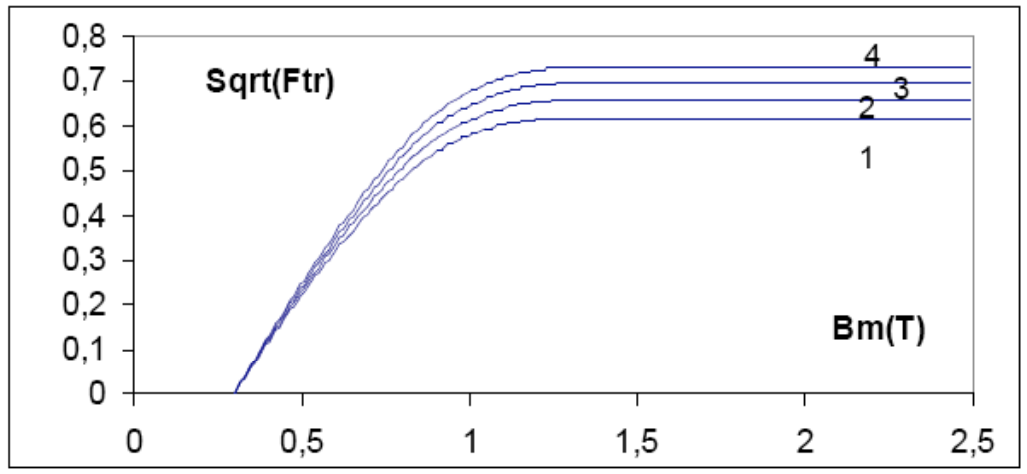

Fig. (28). The influence of the first critical magnetic field $\boldsymbol{B}_{c 1 q}$ of superconducting grains on the trapped magnetic flux Ftr: (1) $\mathrm{B}_{\mathrm{clg}}=100 \mathrm{mT}$, (2) $200 \mathrm{mT}$, (3) $300 \mathrm{mT}$, (4) $400 \mathrm{mT}$.

$\boldsymbol{R}_{g}$ is the radius of the superconducting grain and $\boldsymbol{j}_{c g}$ intragrain current density.

For the next range of the magnetic induction increase determined by the condition $2 \xi \geq B_{m}^{1} \geq \xi$ trapped magnetic flux magnitude, averaged to the cylindrical sample crosssection is equal:

$$
F_{t r}=\frac{2 n B_{\lambda g}}{\xi^{2}}\left(B_{m}^{1} \xi-\frac{\xi^{2}}{2}-\frac{B_{m}^{1}}{4}\right)+\frac{2 \xi}{3}\left(\frac{1}{2}-\left(1-\frac{B_{m}^{1}}{2 \xi}\right)^{3}\right)
$$

For maximum applied magnetic induction in the field cycle, described by the condition $B_{m}^{1} \geq 2 \xi$ the trapped flux reaches the constant value:

$$
F_{t r}=\frac{\xi}{3}+n B_{1 g}
$$

Theoretical results given by Eqs. 32-37 allow then to determine the influence of various material parameters on the flux trapping, which permits then to find the optimum value of these parameters. Examples of calculations of the dependence of the trapping magnetic flux in the cylindrical sample on the maximum magnetic induction amplitude $\boldsymbol{B}_{\boldsymbol{m}}$, versus materials parameters are given in Figs. (26-29) and really indicate on the importance of a given parameter for obtaining maximal flux trapping.

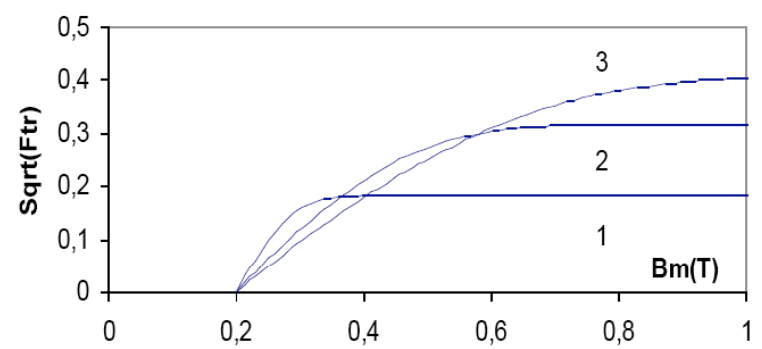

Fig. (29). The influence of the intergranular critical current of the ceramic superconductor on the square root of the flux trapped (Sqrt(Ftr)) versus maximal magnetic field in the cycle. Indices 1-3 indicate to the case of the increasing critical current. 


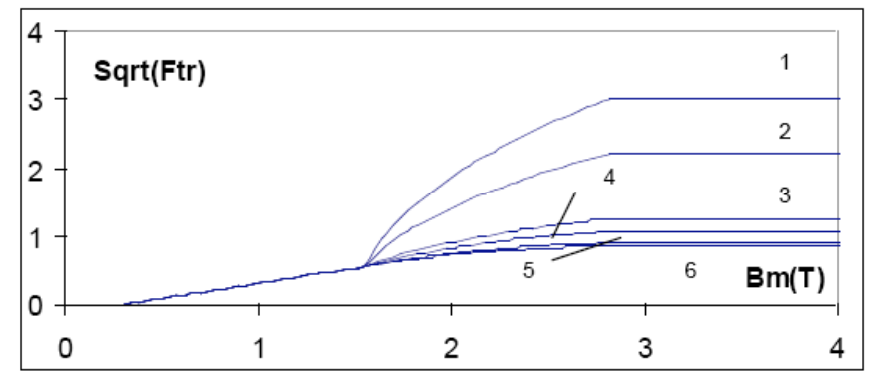

Fig. (30). Dependence of the flux trapping in a superconducting plate on maximum magnetic induction in the cycle $\boldsymbol{B}_{m}$, for various critical current densities inside the superconducting grains. Subsequent curves refer to the following current densities: (1) $\mathrm{j}_{\mathrm{cq}}=10^{12}$, (2) $5^{*} 10^{11}$, (3) $10^{11}$, (4) $5^{*} 10^{10}$, (5) $10^{10}$, (6) $5^{*} 10^{9}$ $\mathrm{A} / \mathrm{m}^{2}$

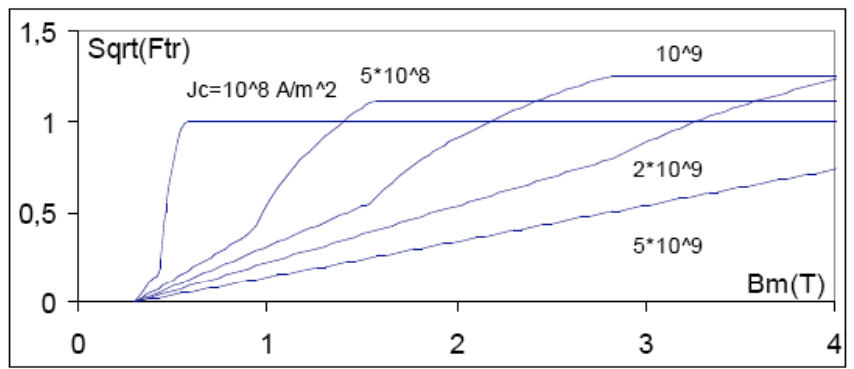

Fig. (31). Dependence of the square root of flux trapping $\boldsymbol{F t r}$ in a superconducting plate on maximum magnetic induction in the cycle $\boldsymbol{B}_{\boldsymbol{m}}$ for various critical current densities inside the superconducting matrix.

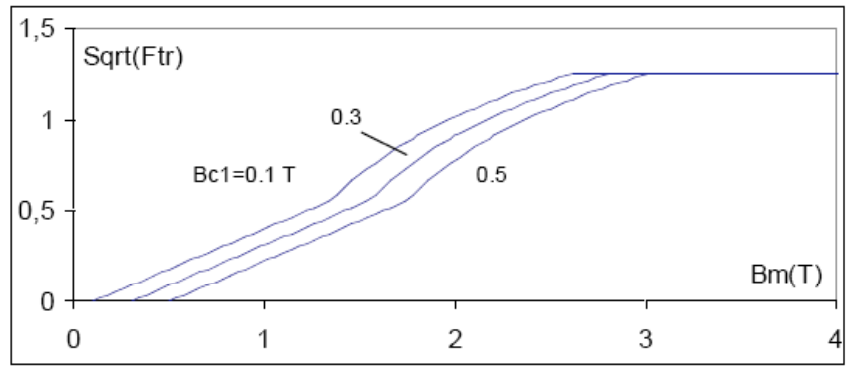

Fig. (32). Dependence of the flux trapping in superconducting plate, on maximum magnetic induction in the cycle $\boldsymbol{B}_{\boldsymbol{m}}$ for various first penetration field $\boldsymbol{B}_{c 1}$

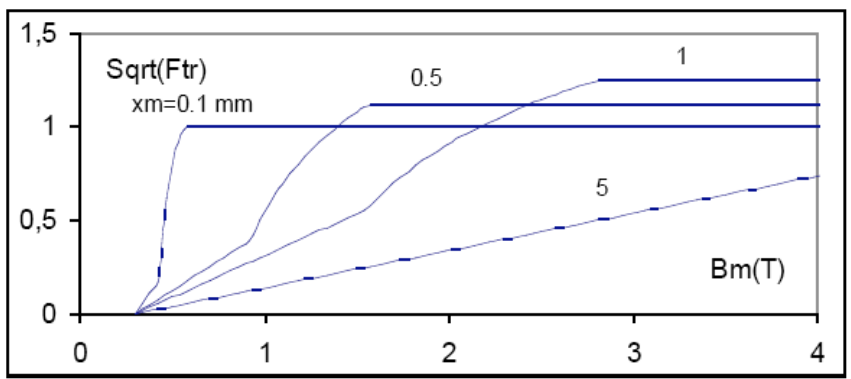

Fig. (33). Dependence of flux trapping in a superconducting plate on maximum magnetic induction in a cycle $\boldsymbol{B}_{\boldsymbol{m}}$ for various flat sample thicknesses $2 \boldsymbol{x}_{\boldsymbol{m}}$.

Beside cylindrical samples described above the case is also significant of the flat ones, which roughly corresponds to the technical tapes and plates. We will analyze now therefore flux trapping for that geometry. As previously four cases dependent on an external magnetic induction amplitude have been considered. The results in each of them are described by Eqs. 38-41, while the graphical presentation of obtained relations is shown in Figs. (30-33).

$$
F_{t r}=0
$$

$$
\begin{aligned}
& F_{t r}=\frac{B_{m}^{2}}{4 B_{p}} \\
& F_{t r}=B_{m}-\frac{B_{m}^{2}}{4 B_{p}}-\frac{B_{p}}{2}+n B_{g}\left(\frac{B_{m}}{B_{p}}-1\right) \\
& F_{t r}=\frac{B_{p}}{2}+n B_{g}
\end{aligned}
$$

$\boldsymbol{B}_{\boldsymbol{m}}$ as previously is the maximum magnetic induction in a field cycle, while $\boldsymbol{B}_{\boldsymbol{p}}$ induction of the full penetration. 


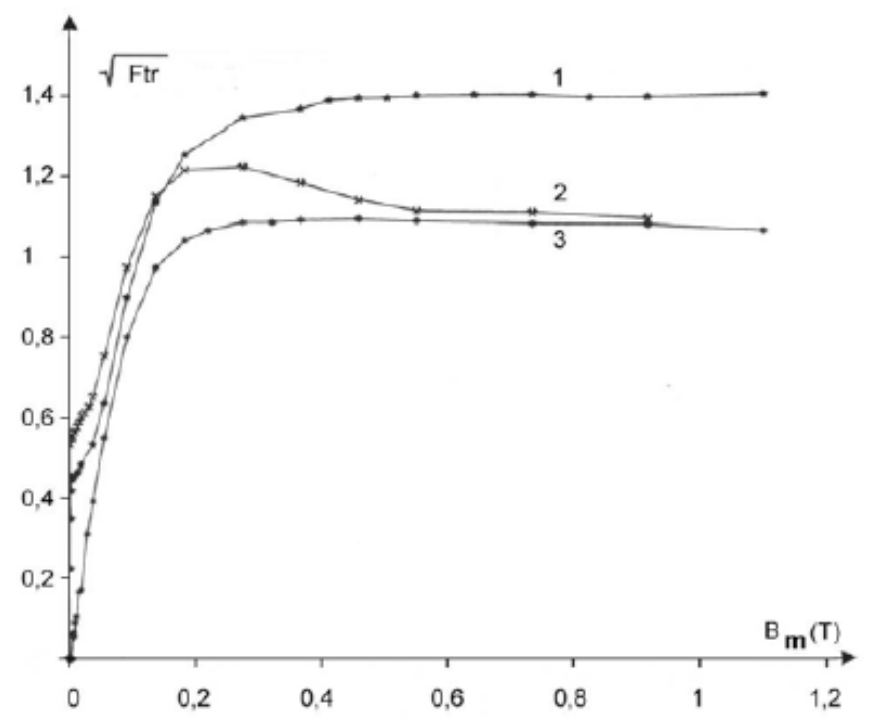

Fig. (34). Experimental dependence of the measured flux trapped versus maximum applied magnetic induction $B_{m}$ for HTc superconducting ceramic of the $\mathrm{YBa}_{2} \mathrm{Cu}_{3} \mathrm{O}_{7-\mathrm{x}}$ type doped with $\mathrm{Fe}$ impurity.

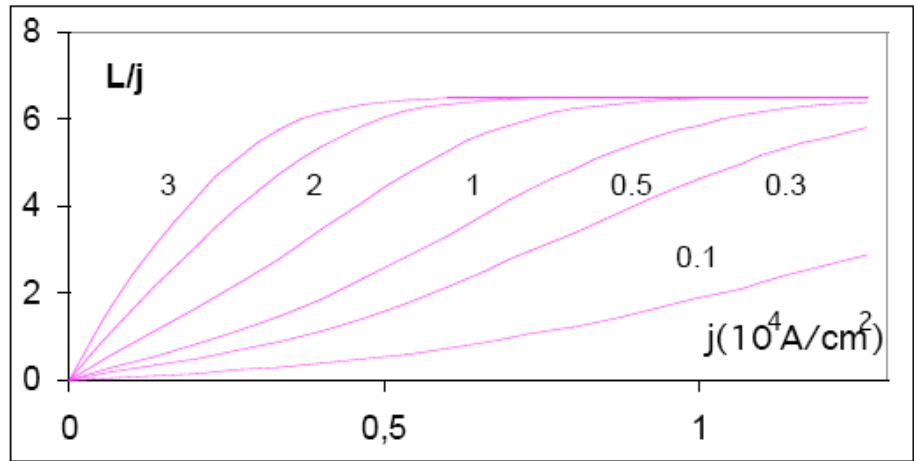

Fig. (35). Calculated a.c. losses in reduced form $L / j$ in a superconducting tape of the second generation $2 \mathrm{G}$, normalised to the transport current density $\boldsymbol{j}$ versus current density, for various values of the parameter $\boldsymbol{\beta}$ given at each curve

The mathematical model presented permits also for taking into account the surface barrier arising on the smooth edge of the superconductor. Then we obtain the following set of equations describing flux trapping in as a function of the maximum external induction amplitude $\mathrm{B}$ defined now according to:

$B_{m}=B_{\max }-B_{c 1}-\Delta B(42)$

In Eq. $42 \mathrm{~B}_{\mathrm{c} 1}$ is the first critical induction, while $\boldsymbol{\Delta B}$ the surface barrier. In the first range of field variation described by the relation:

$B_{c 1}+\Delta B \leq B_{\max } \leq B_{c 1}+2 \Delta B$

the trapped flux is equal to:

$F_{t r}=\frac{j_{c}}{R^{2}}\left[\frac{\left(R+\frac{B_{m}}{j_{c}}\right)^{3}}{3}+R^{2}\left(\frac{B_{m}}{j_{c}}-\frac{R}{3}\right)\right]$

Another relation finds place for magnetic induction within the field range:

$$
\begin{aligned}
& B_{c 1}+2 \Delta B \leq B_{\max } \leq j_{c} R+B_{c 1}+\Delta B \\
& F_{t r}=\frac{1}{6 R^{2} j_{c}}\left[3 R\left(B_{m}{ }^{2}-\Delta B^{2}+2 \Delta B\right)-\frac{\Delta B^{3}+3 B_{m}\left(B_{m} \Delta B+B_{m}{ }^{2}-\Delta B^{2}\right)}{2 j_{c}}\right]
\end{aligned}
$$
tion:

Within the magnetic induction range described by condi-

$$
B_{c 1}+\Delta B+j_{c} R \leq B_{\max } \leq B_{c 1}+\Delta B+2 j_{c} R
$$

appears the relation:

$F_{r r}=\frac{j_{c}}{3 R^{2}}\left[\frac{3 R}{2 j_{c}{ }^{2}}\left(B_{m}{ }^{2}-\Delta B^{2}+2 \Delta B\right)+\left(\frac{B_{m}}{j_{c}}-R\right)^{2}-\frac{\Delta^{3}+3 B_{m}\left(B_{m} \Delta B+B_{m}{ }^{2}-\Delta B^{2}\right)}{4 j_{c}}\right]$

For still higher magnetic field described by:

$2 j_{c} R+B_{c 1}+\Delta B \leq B_{\max }$

the trapped flux reaches constant value:

$F_{t r}=\Delta B+\frac{j_{c} R}{3}$

The presented model allows to follow the influence of superconducting ceramic material parameters on the flux 
trapping, which should be useful in technological problems having aiming at optimalization of this flux and generally of magnetic parameters of the superconductors. On the other hand in this way it is possible from the flux trapping measurements to receive information on such superconducting parameters as critical current, first penetration field and surface barrier. Experimental results of flux trapping measurements performed on $\mathrm{YBa}_{2} \mathrm{Cu}_{3} \mathrm{O}_{7-\mathrm{x}}$ ceramic $\mathrm{Fe}$ doped, according to the reference [8], are shown in Fig. (34) and indicate to a good agreement with the theoretical predictions shown in Figs. (25-33), which independently confirm the theoretical model.

As it has been proved previously, defects and grains boundaries determine the critical current in HTc superconductors and their magnetic properties. Alternating current flow, on the other hand leads to the generation of losses in HTc tapes. Beside pure hysteresis type losses in the modern $2 \mathrm{G}$ tapes the a.c. losses are enhanced due to the magnetic nickel substrate on which $\mathrm{HTc}$ films of $\mathrm{YBaCuO}$ type are deposited. Magnetic substrate leads to an enhancement of the alternating magnetic field in the superconducting film. An example of the calculations of the influence on a.c. losses $\boldsymbol{L}$ of the magnetic characteristics of the nickel substrate described by the expression $\mathbf{B}=\mathbf{0 . 6 5} \tanh (\boldsymbol{\beta} \cdot \mathbf{H})$, where $\beta$ is material parameter is shown in Fig. (35). Results presented here indicate to an important enhancement of a.c. losses with the parameter $\beta$ describing the shape of the magnetic material characteristics.

\section{CONCLUSIONS}

A new model of the pinning interaction has been presented, allowing to determine current-voltage characteristics, critical current, flux pinning and flux trapping in HTc superconductors. The model is based on a detailed analysis of the interaction pancake type vortices with nano-sized pinning centers. A comparison with experimental data of theoretical results is given and a good agreement has been obtained. Influence of the critical current on a.c. losses in tapes of $2 \mathrm{G}$ type with magnetic substrate is discussed briefly.

\section{ACKNOWLEDGMENTS}

Author is grateful to Drs. Eng. Joanna Warycha and Daniel Gajda for performing scanning electron microscopy analysis and magnetization measurements.

\section{REFERENCES}

[1] Yamada, H.; Yamasaki, H.; Develos-Bagarinao, K.; Nakagawa, Y.; Mawatari, Y.; Nie, J.C.; Obara, H.; Kosaka, S. The pinning property of Bi-2212 single crystals with columnar defects. Supercond. Sci. Technol., 2004, 17(1), 58-64.

[2] Okamura, K.; Kiuchi, M.; Otabe, E.S.; Yasuda, T.; Matsushita, T.; Okayasu, S. Flux pinning centres correlated along the c-axis in PLD-YBCO films. Supercond. Sci. Technol., 2004, 17(2), S20-S24.

[3] Zhao, Y.; Cheng, C.H.; Feng, Y.; Shibata, S.; Koshizuka, N.; Murakami, M. The interpretation of improved flux pinning behaviour and second magnetization peaks observed in overdoped $\mathrm{Cu}$-rich Bi2Sr2CaCu2O8+x single crystals. Supercond. Sci. Technol., 2004, 17(2), S83-S87.

[4] Misko, V.R.; Savel'ev, S.; Rakhmanov, A.L.; Nori, F. Negative differential resistivity in superconductors with periodic arrays of pinning sites. Phys. Rev. B, 2007, 75(2), 24509.

[5] Sosnowski, J. Flux Pinning in HTc Superconductors. Int. J. Condensed Matter, Adv. Mat. Supercond. Res. USA, 2008, 6(3-4), 219251.

[6] Sosnowski, J. Dynamic vortex motion in anisotropic HTc superconductors. Mat. Sci. Poland, 2005, 23(3), 613-623.

[7] Sosnowski, J. The influence of the Pinning Centres Dimensions on the Critical Current in High-Tc superconductors. Acta Physica. Polonica., 2004, 106(5), 767-770.

[8] Sosnowski, J. Critical current problems in HTc superconductors with nanoscale pinning centers. EUCAS 2005 Proceedings, $J$. Physics, Conference Series, 2006, 659-662.

[9] Ginzburg, V.L.; Landau, L.D. On the theory of superconductivity. Sov. Phys. JETP, 1950, 20, 1064.

[10] Koblischka, M.; Sosnowski, J. Temperature-dependent scaling of pinning force data in Bi-based high Tc superconductors. Eur. Phys. J., 2005, B44, 270-280.

[11] Sosnowski, J.; Datskov, V.I. Normal and inverse anomaly of dynamical current-voltage characteristic of high Tc oxide superconductors. Cryogenics, 1993, 33(1), 107-111.

[12] Feigelman, M.V.; Geshkenbein, V.B.; Larkin, A.I.; Vinokur, V.M. Theory of collective flux creep. Phys. Rev. Lett., 1993, 63, 2303.

[13] Larkin, A. I.; Ovchinnikov, Y.N. Pinning in Type II Superconductors. J. Low Temp. Phys. Vol. 34, p. 409, 1979.

\begin{tabular}{lll}
\hline Received: December 28, 2010 & Revised: March 19, 2011 & Accepted: March 25, 2011
\end{tabular}

(C) J. Sosnowski ; Licensee Bentham Open.

This is an open access article licensed under the terms of the Creative Commons Attribution Non-Commercial License (http://creativecommons.org/licenses/by-nc/3.0/) which permits unrestricted, non-commercial use, distribution and reproduction in any medium, provided the work is properly cited. 